\title{
Research on the Space-time Operation of the Space-time XML Data
}

\author{
Shaorong Feng \\ (Xiamen University, Information Science and Technology College, Xiamen, China 361005)
}

Keywords: Data base' space-time data; XML; Space-time operation; XQuer

\begin{abstract}
To get space-time data well under control, the elements of space and time have been introduced into XML data. Different from common data, the space-time operation of the space-time XML data requires taking into consideration the elements of space and time at the same time. To make sure the correct space-time operation for space-time XML data, space-time operation has first been classified from the angle of space-time geometric operation and space-time topology operation in the thesis, then the two operation modes are respectively defined and the judgment methods have been proposed in the form of space-time operation function. In the end, the use method of space-time operation in FLWOR expression of XQuery is also researched. By means of space-time operation function, no new query language is needed for space-time XML data query.
\end{abstract}

\section{Introduction}

Data base technology is one of the most important basic fields in today's world computer application. As an important branch of modern data base, space-time data base has a broad application demands in the fields of geographic information system [1] and environmental information system [2].

Although there has been a large amount of research on space-time data [3][4], the research on the traditional data base remains under such limits as temporal extension[5] and structure [6] which both restrict the effectiveness of the traditional data base storing and querying space-time data. XML as the factual standard for information representation, exchange and query, has a wonderful self-description and expansibility [7]. Therefore, the problems related to XML space-time data has become a new research direction [8][9][10].

Space-time operation is one of the important research contents of space-time XML data management, not only requiring the consideration for the elements of space and time, and also the compatibility with the current XML query language. Space-time operation classification is first researched and defined in the thesis and then their judgment methods are proposed, until in the end, the use method of space-time operation in XQuery is put under research.

\section{Classification of space-time operation}

Space-time operation between the spatio-temporal objects can be divided into space-time topological operation and space-time geometric operation. Among, the returned value of the space-time topological operation is TRUE or FALSE which represents the corresponding space-time topological relation between the spatio-temporal objects, including stDisjoint, stMeet, stOverlap, stIntersects, stContain and stEqual. The retuned value of the geometric operation can be the 
space-time data or space data or time data, including stWhen and stHistory. Fig. 1 is the classification of space-time operation and their definitions.

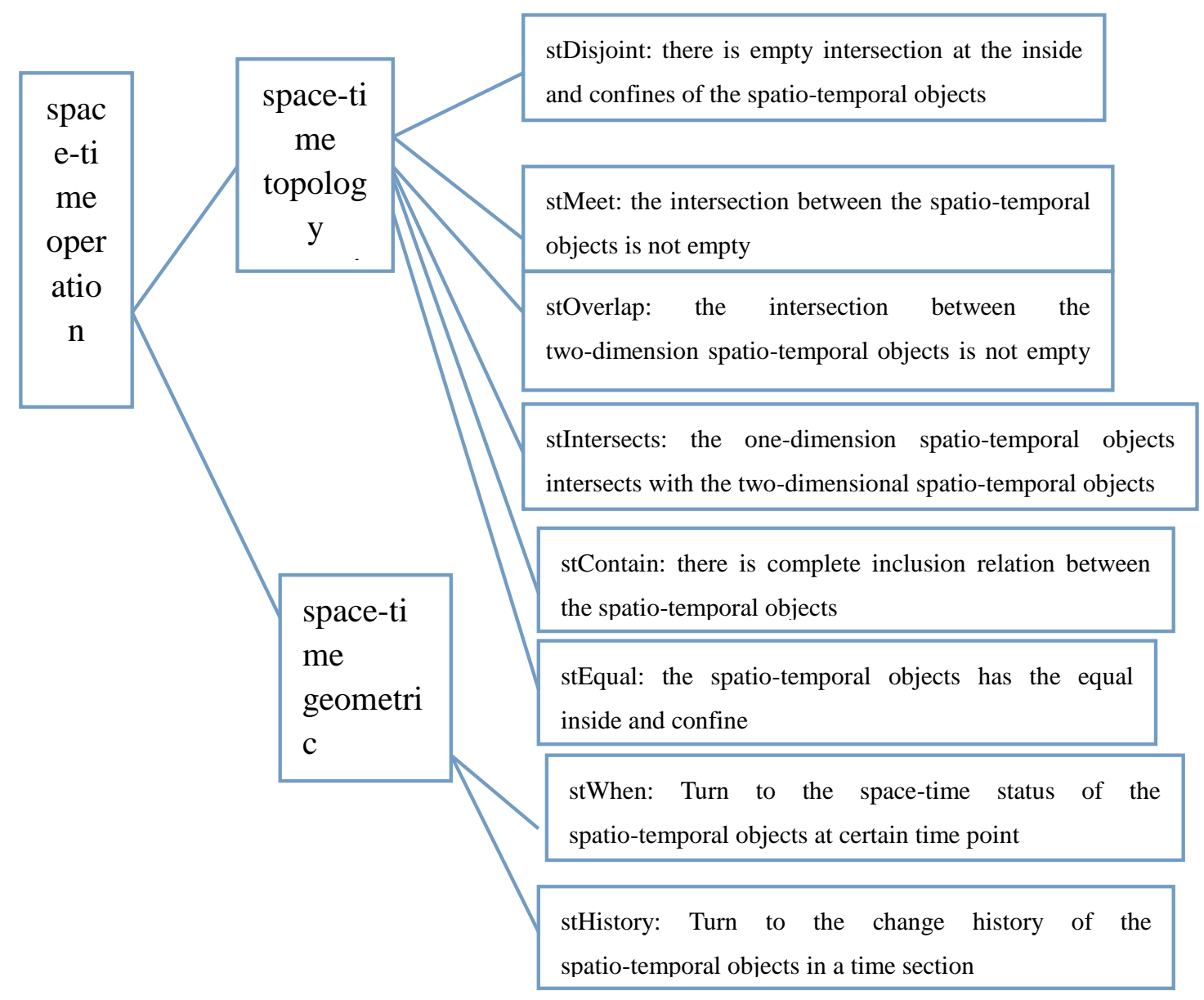

Fig. 1 Categories and meanings of spatiotemporal operations

\section{Space-time operation}

Minimum bounding rectangle (MBR) is adopted in the thesis to represent the spatio-temporal objects, and xl and xr are used to represent MBR. Eight space-time operations will be respectively discussed below, with their definitions first given and then their judgment methods.

Definmition 1 (stDisjoint): in the given time section, the inside and define of the spatio-temporal object SPi both separate from those of the spatio-temporal object SPj, that is called stDisjoint.

Invoking function can be used to judge the stDisjoint topological relation of the spatio-temporal objects, among, function stDisjoint can be defined like below:

define function stDisjoint $(\$ S P i, \$ S P j)$

\{

for \$SPi/@T $\cap \$ S P j / @ T$

if $(\mathrm{SPi} / . . / \mathrm{xr}<\$ \mathrm{SPj} / . . / \mathrm{xl}$ or

$\$ \mathrm{SPi} / . . / \mathrm{yr}<\$ \mathrm{SPj} / . . / \mathrm{yl}$ or

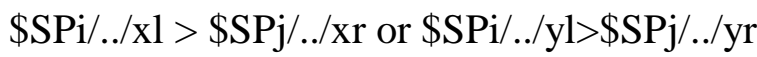

then true ()

else false () 
\}

Definition2 (stMeet): in the given time section, if the confines of the spatio-temporal object SPi and the spatio-temporal object SPj do not have an empty intersection, but their insides have an empty intersection, we can call these two spatio-temporal objects in stMeet.

Invoking function can be used to judge the stMeet topological relation of the spatio-temporal objects, among, function stMeet can be defined like below:

define fuction stMeet $(\$ S P i, \$ S P j)$

\{

for \$SPi /@T $\cap$ \$ SPj /@T

if $(((\$ S P i / . . / x r=\$ \operatorname{SPj} / . . / x 1$ and $(\$ S P i / . . / y l<=\$ S P j / . . / y l<=\$ S P i / . . / y r$ or $\$ S P i / . . / y l<=\$ S P j$ /../yr $<=\$ \mathrm{SPi} / . . / \mathrm{yr}))$ or

$(\$ \operatorname{SPi} / . . / \mathrm{yr}=\$ \mathrm{SPj} / . . / \mathrm{yl}$ and $(\$ \mathrm{SPi} / . . / \mathrm{xl}<=\$ \mathrm{SPj} / . . / \mathrm{xl}<=\$ \mathrm{SPi} / . . / \mathrm{xr}$ or $\$ \mathrm{SPi} / . . / \mathrm{xl}<=\$ \mathrm{SPj} / . . / \mathrm{xr}<=$ $\$ \mathrm{SPi} / . . / \mathrm{xr}))$ or

$(\$ S P i / . . / x l=\$ S P j / . . / x r$ and $(\$ S P i / . . / y l<=\$ S P j / . . / y l<=\$ S P i / . . / y r$ or $\$ S P i / . . / y l<=\$ S P j / . . / y r<=$ $\$ \mathrm{SPi} / . . / \mathrm{yr}))$ or

(\$SPi /../yl = \$SPj /../yr and (\$SPi /../xl <= \$SPj /../xr <=\$ SPi /../xr or \$SPi /../xl $<=\$ S P j / . . / x l$ $<=\$ \mathrm{SPi} / . . / \mathrm{xr})))$

then true ()

else false ()

\}

Definition3 (stOverlap): in the given time section, two-dimension spatio-temporal object SPi and the two-dimension spatio-temporal object SPj partly overlap and the overlap section is also two-dimension, these two spatio-temporal objects will be in stOverlap relation.

Invoking function can be used to judge the stOverlap topological relation of the spatio-temporal objects, among, function stOverlap can be defined like below:

define fuction stOverlap (\$SPi, $\$ \mathrm{SPj})$

\{

for $\$ \mathrm{SPi} / @ \mathrm{~T} \cap \$ \mathrm{SPj} / @ \mathrm{~T}$

if $(((\$ \mathrm{SPi} / . . / \mathrm{xl}<=\$ \mathrm{SPj} / . . / \mathrm{xr}$ and $\$ \mathrm{SPi} / . . / \mathrm{yl}<=\$ \mathrm{SPj} / . . / \mathrm{yr})$ and $\$ \mathrm{SPi} / . . / \mathrm{xl}>\$ \mathrm{SPj} / . . / \mathrm{xl})$ or

$((\$ \mathrm{SPi} / . . / \mathrm{xr}>=\$ \mathrm{SPj} / . . / \mathrm{xl}$ and $\$ \mathrm{SPi} / . . / \mathrm{yl}<=\$ \mathrm{SPj} / . . \mathrm{yr})$ and $\$ \mathrm{SPi} / . . / \mathrm{xl}>\$ \mathrm{SPj} / . . / \mathrm{xl})$ or

$((\$ \mathrm{SPi} / . . / \mathrm{xr}>=\$ \mathrm{SPj} / . . / \mathrm{xl}$ and $\$ \mathrm{SPi} / . . / \mathrm{yl}<=\$ \mathrm{SPj} / . . / \mathrm{yr}) \$ \mathrm{SPi} / . . / \mathrm{xr}<\$ \mathrm{SPj} / . . / \mathrm{xr}$ ) or

$((\$ S P i / . . / x r>=\$ S P j / . . / x r$ and $\$ S P i / . . y r ~>=\$ S P j / . . / y l)$ and $\$ S P i / . . / x r<\$ S P j / . . / x r))$

then true ()

else false ()

\}

When a one-dimension spatio-temporal object partly overlaps with a two-dimension spatio-temporal object, they can be defined in stIntersects relation. Because MBR is used in the thesis to describe two-dimension spatio-temporal object, and the one-dimension spatio-temporal object can be seen as a special two-dimension spatio-temporal object, in sense, it can be said that stIntersects is a special case of stOverlap.

Definition4 (stContain): in the given time section, spatio-temporal object SPi is completely contained inside of spatio-temporal object SPj, we can judge their stContain relation.

Invoking function can be used to judge the stContain topological relation of the spatio-temporal objects, among, function stContain can be defined like below: 
define function stContain $(\$ \mathrm{SPi}, \$ \mathrm{SPj})$

\{

for \$SPi/@T $\cap \$ S P j / @ T$

if $(\$ S P i / . . / x 1<\$ S P j / . / x l$ and $\$ S P i / . / y l<\$ S P j / . . / y l)$ and

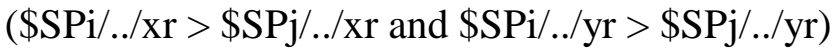

then true ()

else false ()

\}

Definition 5 (stEqual): in the given time section, when spatio-temporal object SPi has an equal inside and confines to spatio-temporal object $\mathrm{SPj}$, these two spatio-temporal objects will be in the stEqual relation.

Invoking function can be used to judge the stEqual topological relation of the spatio-temporal objects, among, function stEqual can be defined like below:

define function stEqual $(\$ S P i, \$ S P j)$

\{

for \$SPi/@T $\cap \$ S P j / @ T$

if $(\$ S P i / . . / x l=\$ S P j / . . / x l$ and $\$ S P i / . . / y l=\$ S P j / . . / y l)$ and

$(\$ S P i / . . / x r=\$ S P j / . . / x r$ and $\$ S P i / . . / y r=\$ S P j / . . / y r)$

then true ()

else false ()

\}

In a sense, stWhen is something like snapshot, which explains it is another named space-time snapshot.

Definition 6 (stWhen): in the given time section, stWhen returns to spatial status of the spatio-temporal object at the time point.

Invoking function can be used to get the space-time attribute status of the spatio-temporal object at a given time point, among, function stWhen can be defined like below:

define function stWhen $(\$ \mathrm{~m}, \$ \mathrm{t})$

\{

for $\$ \mathrm{~m}$

if $\$ \mathrm{SP} / . . /$ motion $=\varnothing$

if $\$ \mathrm{~m} / @$ tstart $<\mathrm{t}<\$ \mathrm{~m} /$ @ tend

return $\$ \mathrm{~m}$ where $\$ \mathrm{~m} / \mathrm{tstar}=$ tend=t

if $\$ S P / . . /$ motion $^{1} \varnothing$

return $\$ \mathrm{~m}$

if $\mathrm{m}=\mathrm{OID}$

return $\mathrm{m} / \mathrm{type}, \mathrm{m}$ /predecessor, $\mathrm{m} /$ successor

where $\$ \mathrm{~m} /$ tstart=tend $=\mathrm{t}$

\}

stHistory can query the change history of the spatio-temporal object in a given time section.

Definition 7 (stHistory): in a given time section, stHistory return to the change history of the spatio-temporal object in the time section.

Invoking function can be used to get the space-time attribute status of the spatio-temporal object in a given time section, among, function stHistory can be defined like below: 


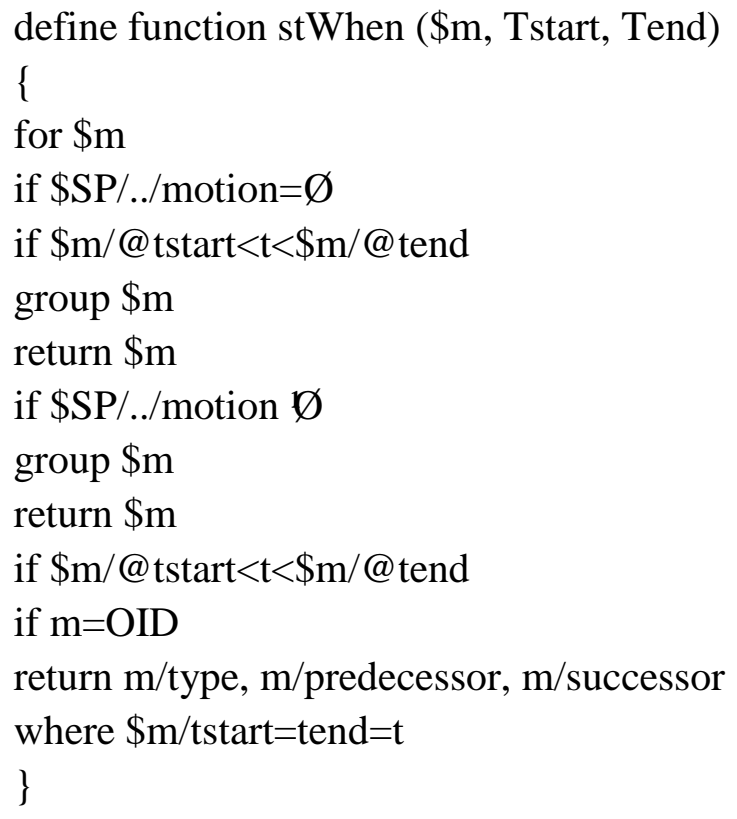

A group function is used in stHistory calculation method which can return all the different results.

\section{The application of space-time operation in XQuery}

Eight space-time functions have been introduced for the concrete space-time operation: stDisjoint, stMeet, stOverlap, stIntersects, stContain, stEqual, stWhen and stHistory. Among, each function has its corresponding parameter. Therefore, it only requires invoking the corresponding time-space functions to use XQuery for space-time operation in XML, and the space-time function can be corresponded to XML nodes through parameters.

Return Funtion. In FLWOR expression, the uses and expressions of for, let, where and order by can be directly used like while common data query. The query results in the thesis are mainly specific to the space-time operation, so it only needs to bind the defined space-time function behind the return to acquire the desired space-time operation result. The part of return can be expressed in the form as below:

Return Funtion. Among, Function means space-time operation function, include stDisjoint, stMeet, stOverlap, stIntersects, stContain, stEqual, stWhen and stHistory. Therefore, when using XQuery for query, FLWOR can be used for expression, the use methods and expressions of for, let, where, order by can keep unchanged, and it only needs to bind space-time function in the part of return.

For example, stDisjoint function can be invoked in XQuery to get to know if two spatio-temporal objects are in stDisjoint topological relation, if the TRUE is returned, it proves stDisjoint topological relation; if the returned value is FALSE, they are not in the stDisjoint relation.

\section{Conclusions}

The classification of the space-time operation has first been explored in the thesis, by which it can be classified stDisjoint, stMeet, stOverlap, stIntersects, stContain, stEqual, stWhen and stHistory, and then their definitions are respectively given. On top of this, their judgment methods are also proposed in the form of space-time operation function. In the end, the research shifts to the use 
method of FLWOR expression in XQuery. By means of space-time operation function, no new query language is needed for space-time XML data query.

\section{Acknowledgement}

The thesis has been kindly sponsored by such institutes as National Natural Science Foundation of China (NSFC) (No.61303004), the national social science fund major projects (No.13\&ZD148); Natural science fund project in Fujian Province (No.2013J05099)

\section{References}

[1] Deng S S, Xia L H, Wang F. Analysis of spatio-temporal characteristics of urban land cover and its landscape pattern: a case study in Nanhai district of Foshan city [J]. Urban Remote Sensing Event, 2009, 22(20): 1-9.

[2] Mehrotra R, Sharma A. Evaluating spatio-temporal representations in daily rainfall sequences from three stochastic multi-site weather generation approaches [J]. Advances in Water Resources, 2009, 32(6): 948-962.

[3] Pelekis N, Theodoulidis B, Kopanakis I, et al. Literature review of spatio-temporal database models [J]. The Knowledge Engineering Review, 2004, 19 (3): 235-274.

[4] Nandal R. Spatio-temporal database and its models: a review [J]. Journal of Computer Engineering, 2013, 11 (2): 91-100.

[5] Pfoser D, Tryfona N. Requirements, definitions, and notations for spatiotemporal application environments [C]//Proceedings of the 6th ACM Symposium on GIS. 1998: 124-130.

[6] Rizzolo F, Vaisman A. A. Temporal XML: modeling, indexing, and query processing [J]. International Journal on Very Large Data Bases, 2008, 17(5): 1179-1212.

[7] Senellart S, Abiteboul S. On the complexity of managing probabilistic XML data $[C] / /$ Proceedings of the 26th ACM SIGACT-SIGMOD-SIGART Symposium on Principles of Database Systems. 2007: 283-292.

[8] Jiang X. J, Zhang Y. P, Pi D. C. An XML-based data model for moving object database [J]. Communications in Computer and Information Science, 2010, 118: 19-28.

[9] Franceschet M, Montanari A, Gubiani D. Modeling and validating spatio-temporal conceptual schemas in XML Schema [C]//Proceedings of the 18th International Conference on Database and Expert Systems Applications (DEXA 2007). 2007: 25-29.

[10]Liu X. H, Wan Y. C. Storing spatio-temporal data in XML native database [C]//Proceedings of the 2nd International Workshop on Database Technology and Applications. 2010: 1-4. 